Nagnet ohydr odynami c si mul at i on on co- and count er-hel i ci ty mergi ng of spher omaks and dr i ven magnet i c reconnect i on

\begin{tabular}{|l|l|}
\hline $\begin{array}{l}\text { j our nal or } \\
\text { publ i cat i on ti t l e }\end{array}$ & Physi cs of pl asmas \\
\hline vol une & Vol. 4 \\
\hline number & No. 5 \\
\hline page r ange & pp. 1297- 1307 \\
\hline year & 1997- 05- 01 \\
\hline URL & ht t p: //hdl . handl e. net /10655/2284 \\
\hline
\end{tabular}




\title{
Magnetohydrodynamic simulation on co- and counter-helicity merging of spheromaks and driven magnetic reconnection
}

\author{
T.-H. Watanabe, T. Sato, and T. Hayashi \\ Theory and Computer Simulation Center, National Institute for Fusion Science, Nagoya 464-01, Japan
}

(Received 30 July 1996; accepted 10 February 1997)

\begin{abstract}
A magnetohydrodynamic relaxation process of spheromak merging is studied by means of an axisymmetric numerical simulation. As a result of counter-helicity merging, a field-reversed configuration is obtained in the final state, while a larger spheromak is formed after co-helicity merging. In the counter-helicity case, a clear pressure profile of which iso-surfaces coincide with flux surfaces is generated by thermal transport of a poloidal flow induced by driven reconnection. It is also found that a sharp pressure gradient formed in the vicinity of a reconnection point causes a bouncing motion of spheromaks. According to the bounce motion, the reconnection rate changes repeatedly. As shown by the Tokyo University Spherical Torus No. 3 (TS-3) experiments [M. Yamada, et al., Phys. Rev. Lett. 65, 721 (1990)], furthermore, strong acceleration of a toroidal flow and reversal of a toroidal field in the counter-helicity merging were observed. (C) 1997 American Institute of Physics. [S1070-664X(97)02905-4]
\end{abstract}

\section{INTRODUCTION}

The coalescence process of spheromaks has been experimentally studied using the Tokyo University Spherical Torus No. 3 (TS-3) device. ${ }^{1-5}$ In the TS-3 experiments, it is shown that a FRC (field-reversed configuration) is formed after counter-helicity merging where two spheromaks have antiparallel magnetic helicity, while a larger spheromak is obtained after co-helicity merging of spheromaks with the same helicity. In the counter-helicity case, driven reconnection causes strong heating and acceleration of ions and reversal of the toroidal field. The counter-helicity merging gives not only a potential method of slow formation of the FRC, but also a remarkable example for studying magnetic reconnection and MHD (magnetohydrodynamic) relaxation with finite pressure. It is noteworthy that the formation of pressure profile in the FRC cannot be explained by Taylor's relaxation theory ${ }^{6,7}$ where a zero- $\beta$ plasma (or a uniform pressure profile) is assumed.

The first MHD simulation on the spheromak merging was carried out more than ten years ago for the co-helicity case in the zero- $\beta$ limit. ${ }^{8}$ In the co-helicity merging, a larger spheromak is formed through the driven reconnection. The simulation result agrees well with Taylor's theory. Recently, we made preliminary simulation studies on the counterhelicity merging in an axisymmetric system, and confirmed that the final state in the counter-helicity case was the FRC. ${ }^{9,10}$ Nevertheless, dynamic processes of spheromak merging have not yet been fully revealed.

In this paper, we will study the co- and counter-helicity merging of spheromaks using an axisymmetric MHD simulation code with high spatial resolution. We will focus our attention to find how the plasma pressure is confined in the FRC in the counter-helicity case, and how the spheromak merging with a finite- $\beta$ effect develops through the driven magnetic reconnection. Comparison of the simulation results with the experimental evidence will help us to understand physical processes of spheromak merging.

We will show our simulation model in the next section.
Overviews of simulation results for the counter- and cohelicity cases are given in Sections III A and B, respectively. In Sections III C to E, we will present more detailed results, i.e., finite-pressure effects on magnetic reconnection, spontaneous confinement of pressure in the FRC, and toroidal oscillation of a reconnected magnetic loop. We have also investigated effects of parallel thermal conduction on the spheromak merging in Section III F, using a newly developed simulation code with inhomogeneous grid spacing. The results are summarized in the last section.

\section{SIMULATION MODEL}

To study merging processes of spheromaks, we solve the MHD equations for a finite- $\beta$ plasma. The governing equations are as follows:

$$
\begin{aligned}
& \rho \frac{d \mathbf{v}}{d t}=-\nabla p+\mathbf{j} \times \mathbf{B}+\mu\left(\nabla^{2} \mathbf{v}+\frac{1}{3} \boldsymbol{\nabla}(\boldsymbol{\nabla} \cdot \mathbf{v})\right), \\
& \frac{1}{(\gamma-1)} \frac{d p}{d t}=-\frac{\gamma}{\gamma-1} p \boldsymbol{\nabla} \cdot \mathbf{v}+\eta \mathbf{j}^{2}+\Phi,
\end{aligned}
$$

and

$$
\frac{\partial \mathbf{B}}{\partial t}=-\boldsymbol{\nabla} \times \mathbf{E} .
$$

Here, $\mathbf{j}, \mathbf{E}, \Phi$, and $e_{i j}$ are given by

$$
\begin{aligned}
& \mathbf{j}=\boldsymbol{\nabla} \times \mathbf{B}, \\
& \mathbf{E}=-\mathbf{v} \times \mathbf{B}+\eta \mathbf{j}, \\
& \Phi=2 \mu\left(e_{i j} e_{i j}-\frac{1}{3}(\boldsymbol{\nabla} \cdot \mathbf{v})^{2}\right),
\end{aligned}
$$

and

$$
e_{i j}=\frac{1}{2}\left(\frac{\partial v_{i}}{\partial x_{j}}+\frac{\partial v_{j}}{\partial x_{i}}\right) .
$$

$\gamma, \mu$, and $\eta$ are ratio of the specific heats $(\gamma=5 / 3)$, viscosity $\left(\mu=1 \times 10^{-4}\right)$, and resistivity $\left(\eta=1 \times 10^{-4}\right)$. In simulation 


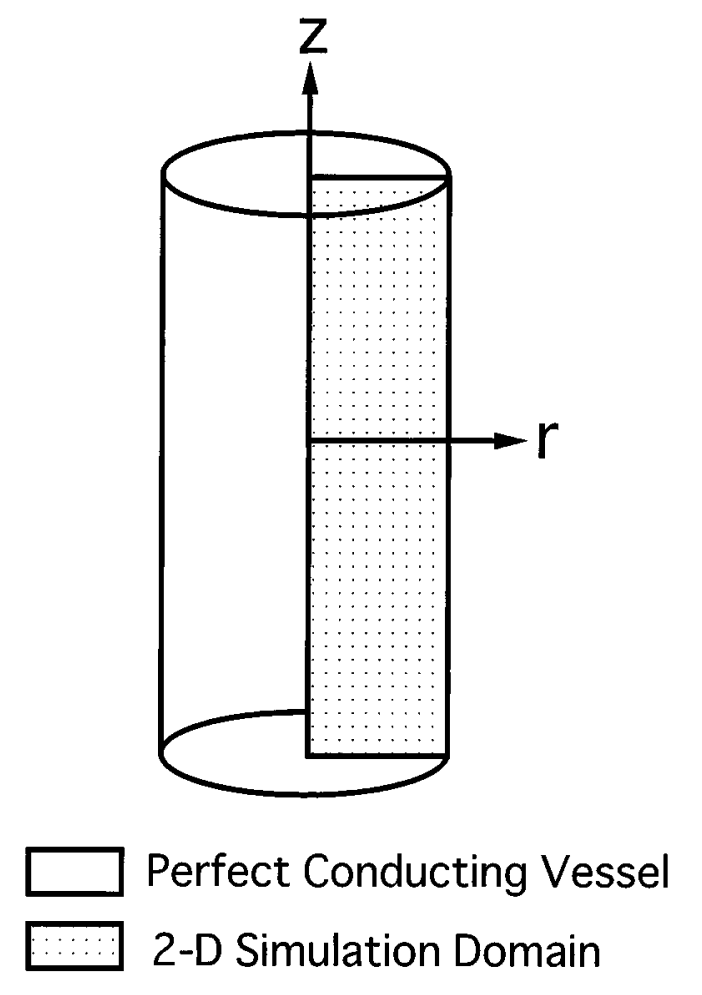

FIG. 1. Schematic plot of the simulation system in cylindrical coordinates $(r, \varphi, z)$. Shaded poloidal plane at $\varphi=0$ shows the two-dimensional simulation domain. Perfect conducting boundary is located at $r=1$ and $z= \pm 2$. At $t=0$, two spheromaks with radius of $a=0.5$ are placed at $z= \pm 0.75$.

runs given in Section III C, we will change the value of $\gamma$ as a physical parameter. $\Phi$ is a dissipation function due to the viscosity; $e_{i j}$ denotes a rate-of-strain tensor. Other notations are standard. All of the physical quantities are normalized by typical Alfvén speed $V_{\mathrm{A}}$, mass density $\rho$, and radius of a cylindrical vessel $R_{V}$. Here, $\rho$ is assumed to be uniform and constant in time for simplicity. Time-integration of $\mathbf{B}, \mathbf{v}$, and $p$ is calculated by the fourth-order Runge-Kutta-Gill method. Spatial derivatives are computed by the secondorder finite difference.

The present simulation system is schematically plotted in Fig. 1. The cylindrical vessel is set in $0<r<1$ and $-2<z<2$. We assume the axisymmetry, and carry out numerical simulations in a two-dimensional poloidal plane which is shown by shade in Fig. 1. The simulation domain is represented by spatial grid points of $289 \times 1153$ in $(r, z)$ coordinates. To check convergence against the grid spacing, we have also performed a simulation using finer grid points of $577 \times 2305$, and have obtained the same results with what will be shown in this paper. We use a perfect conducting boundary at the vessel wall. Velocity is fixed to zero on the boundary, while pressure is calculated so that no thermal flux passes through the wall. Initially, a homogeneous zero- $\beta$ plasma fills the simulation domain, such as $\rho=1, p=0$, and $\mathbf{v}=0$. An initial condition for magnetic field is given as follows. At first we set a pair of toroidal currents $j_{\varphi}$ centered at $z= \pm 0.75$. Each of the toroidal current patterns is given by the Hill's vortex solution ${ }^{11}$ of $\boldsymbol{\nabla} \times \mathbf{B}=\lambda \mathbf{B}(\lambda=$ constant $)$ with its radius of $a=0.5$. Directions of the toroidal current are set

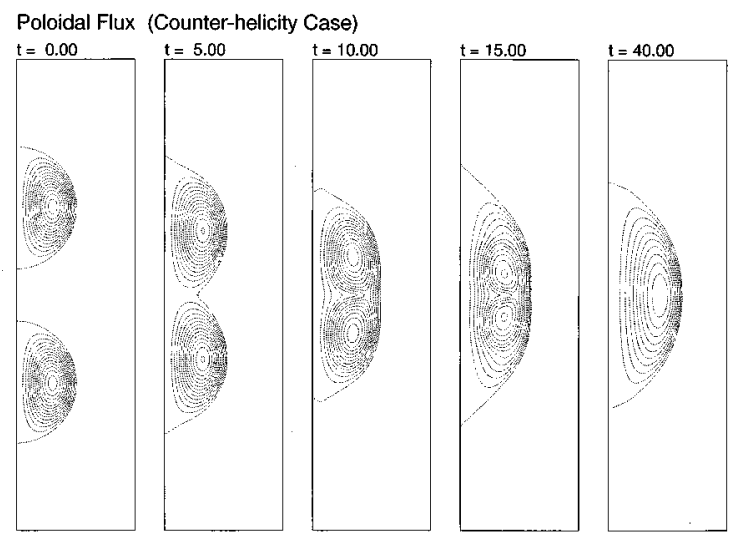

FIG. 2. Contours of the poloidal flux at different time steps for merging of two spheromaks with anti-parallel helicity. Only negative parts are plotted for clarity. Contour interval is set to be $2.5 \times 10^{-3}$.

to be the same (negative $\varphi$ direction) in order to make the spheromaks merge. Second, to determine $B_{r}$ and $B_{z}$ consistently with $j_{\varphi}$, we numerically solve

$$
-\nabla^{2} A_{\varphi}+A_{\varphi} / r^{2}=j_{\varphi}
$$

where $A_{\varphi}$ means $\varphi$ component of the vector potential. A boundary condition for $A_{\varphi}$ is

$$
\frac{1}{r} \frac{\partial}{\partial r}\left(r A_{\varphi}\right)=B_{z}=-\frac{2 C}{x_{1}} \sin x_{1} \text { at } r=1,
$$

and

$$
-\frac{\partial A_{\varphi}}{\partial z}=B_{r}=0 \quad \text { at } \quad z= \pm 2 .
$$

Here, $x_{1}$ denotes the first zero of the first-order spherical Bessel function, that is, $x_{1}=4.493$, and we set the constant $C=2.5$. Finally, the toroidal field $B_{\varphi}$ is given by

$$
B_{\varphi}= \pm C x_{1} A_{\varphi} / a \text { for } A_{\varphi} \leqslant 0
$$

or

$$
B_{\varphi}=0 \text { for } A_{\varphi}>0 .
$$

Each of $B_{\varphi}$ in the two spheromaks has different signs in the counter-helicity case but has the same negative sign in the co-helicity case. The maximum intensity of $B_{\varphi}$ at $t=0$ is about 1.1. The initial configuration of the magnetic field does not satisfy the force-free condition, although the current density in perpendicular to the magnetic field is much weaker than the parallel one. This is because each of the spheromaks feels the poloidal field of the other one. Then, the spheromaks attract each other by the Lorentz force and start to merge at $z=0$.

\section{SIMULATION RESULTS}

\section{A. An overview of counter-helicity merging}

Here, we present an overview of simulation results for the counter-helicity merging of spheromaks. Contours of the poloidal flux $\Psi$ at different time steps are shown in Fig. 2, where only negative regions are plotted in the poloidal plane. The outermost line corresponds to $\Psi=0$. Thus, separatrixes 


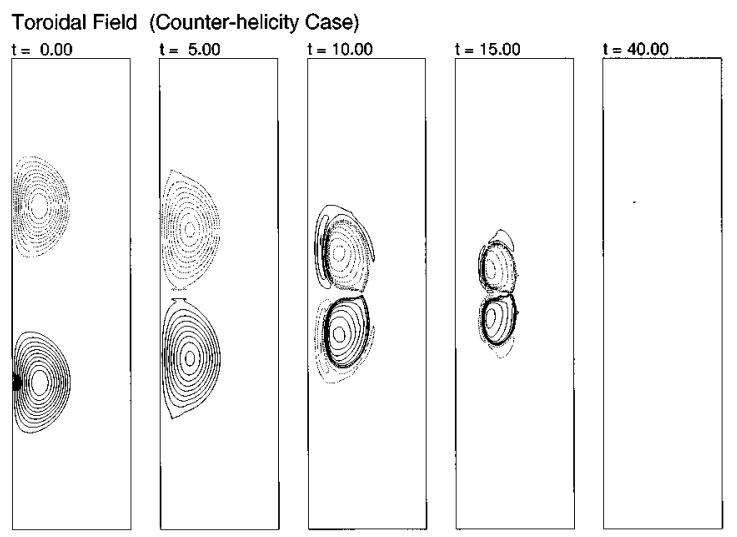

FIG. 3. Same as Fig. 2 but for the toroidal field contours with interval of 0.1 . Solid and dotted lines show positive and negative components, respectively.

locate at cross-points of $r=0$ and $\Psi=0$. In the initial stage of simulation $\left(t<5 \tau_{\mathrm{A}} ; \tau_{\mathrm{A}}\right.$ denotes Alfvén transit time), one can see that the two spheromaks approach the $z=0$ line. Then, the driven magnetic reconnection ${ }^{12}$ takes place at $z=0$, where a reconnection point (X-point defined as a saddle point of the poloidal flux contour) and a current sheet are formed between the two spheromaks. As time goes on, the reconnection proceeds deeper into the unmerged region, and the reconnected flux increases. The merging of spheromaks has been completed at $t=25 \tau_{\mathrm{A}}$.

The toroidal magnetic field is plotted in Fig. 3 by solid and dashed contours. As the merging continues, the antiparallel toroidal field annihilates and finally disappears. Thus, a large amount of toroidal field energy is released in the counter-helicity merging. The final configuration without mean toroidal field is of FRC type. In addition, one can see that the toroidal field is reversed around unmerged flux surfaces (see $t=10$ and $15 \tau_{\mathrm{A}}$ ). The reversal of the toroidal field is observed also in the TS-3 experiments and is considered to be closely related with a toroidal flow. ${ }^{1,4}$ A physical mechanism of the field reversal will be elucidated in Section III E.

Figure 4 shows pressure profiles at different time steps. In the merging phase $\left(t=10\right.$ and $\left.15 \tau_{\mathrm{A}}\right)$ the pressure peaks at $z=0$, where the reconnection takes place and the magnetic

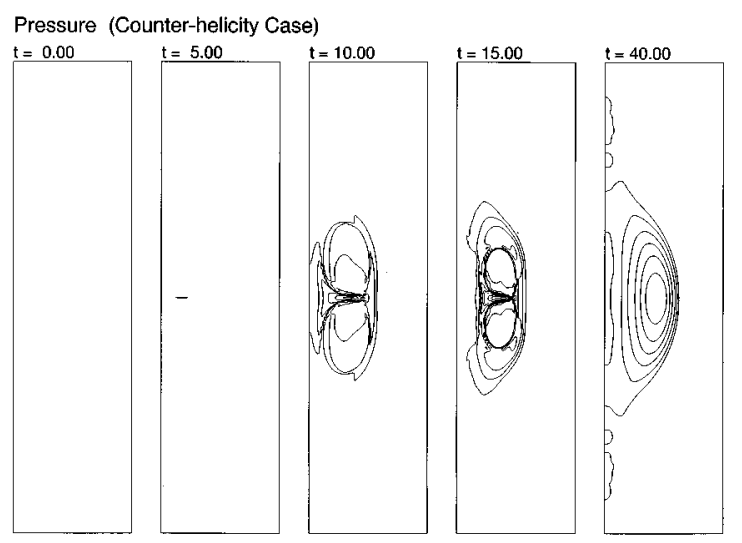

FIG. 4. Same as Fig. 2 but for the pressure contours with interval of $2.5 \times 10^{-2}$

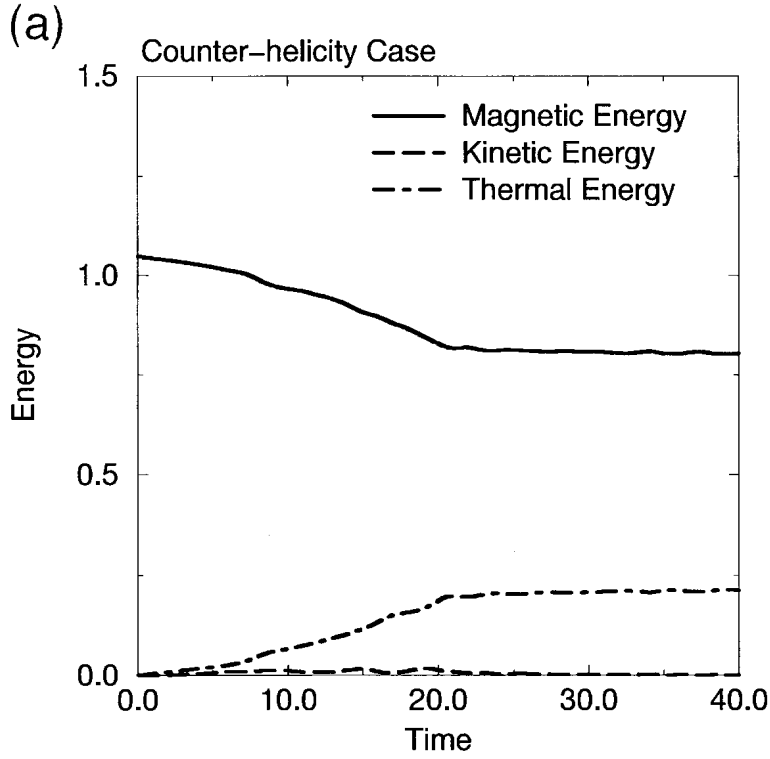

(b)
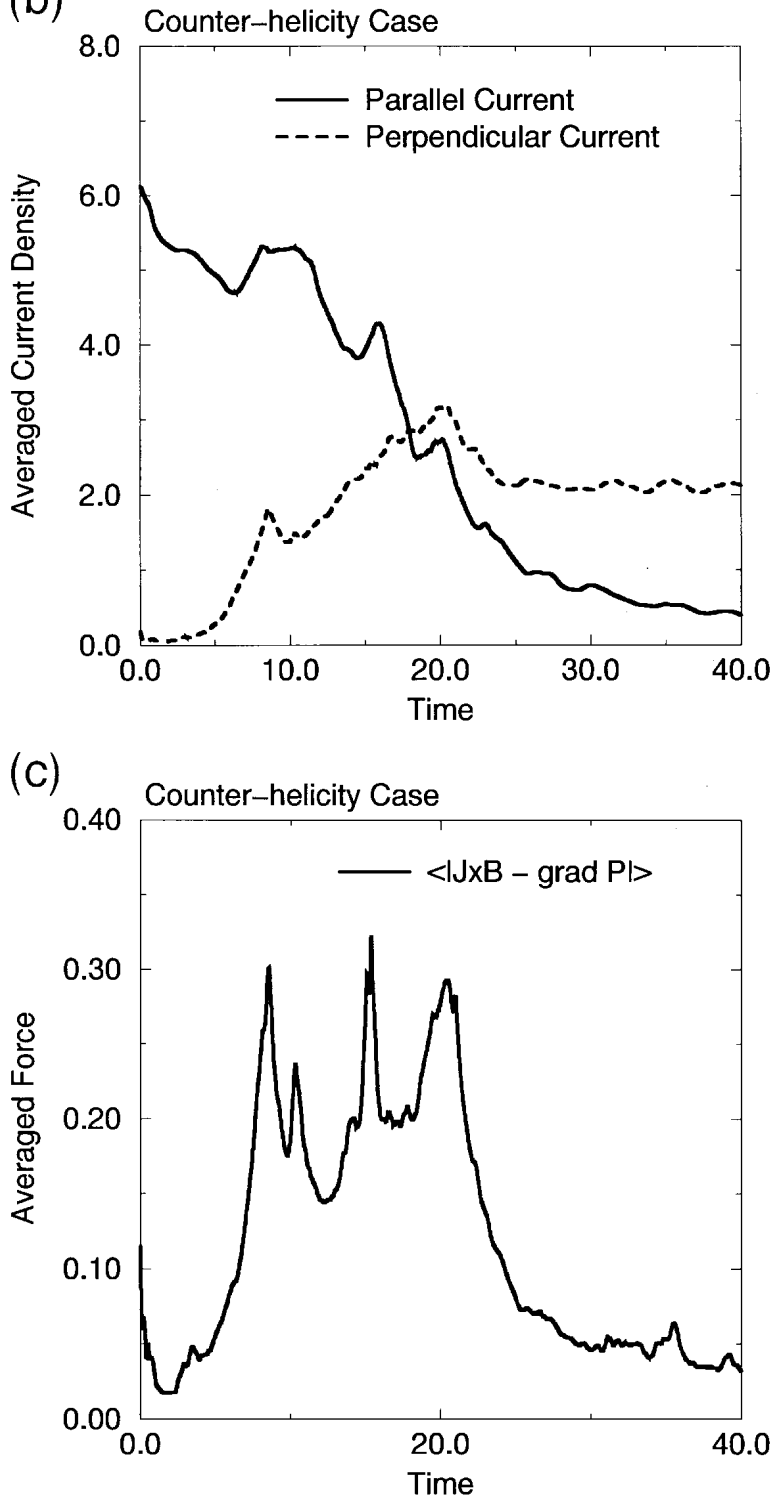

FIG. 5. Time evolutions of (a) energies in the system, (b) the parallel and perpendicular currents, and (c) the spatially averaged force. 


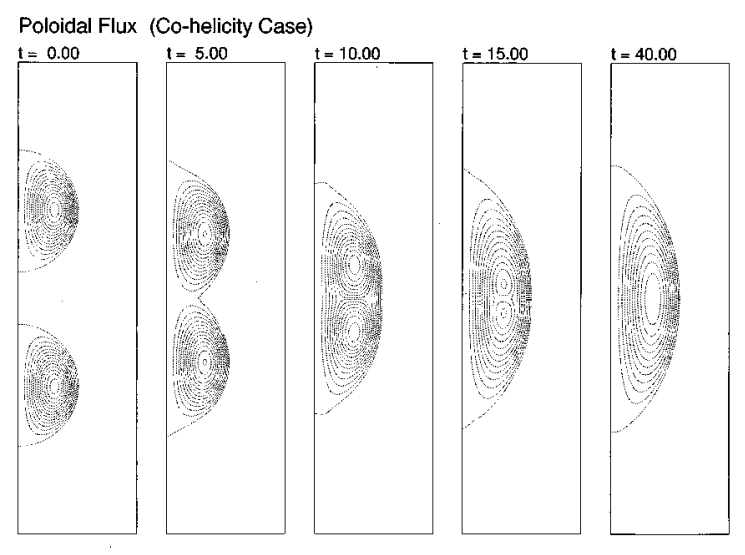

FIG. 6. Same as Fig. 2 but for the co-helicity case.

energy is converted to the thermal energy. The increased pressure profile is broadened into the system along the reconnected flux surfaces. A clear pressure profile has been formed after merging, though the thermal conduction effect is not taken into account. In the final state, the iso-pressure surfaces coincide with the flux surfaces, in other words, the poloidal field supports the pressure gradient. Furthermore, it should be noted that most of the increased pressure is confined in the FRC.

Temporal plots of magnetic, kinetic, and thermal energies in the cylindrical vessel are shown in Fig. 5(a). About $20 \%$ of the initial magnetic energy decreases in the counterhelicity merging from $t=7$ to $20 \tau_{\mathrm{A}}$. Most of the released magnetic energy is converted to the thermal energy. After $t=20 \tau_{\mathrm{A}}$, all of the energies are nearly constant, and the system approaches to a steady state. One can find three faint peaks in the time evolution of the kinetic energy (at $t=9$, 15 , and $19 \tau_{\mathrm{A}}$ ). It means that acceleration of a plasma flow, namely, the driven reconnection is enhanced three times, repeatedly. Detailed results found in the time evolution of the driven reconnection are given in Section III C.

In Fig. 5(b), we have plotted the parallel and perpendicular components of current density averaged inside of the

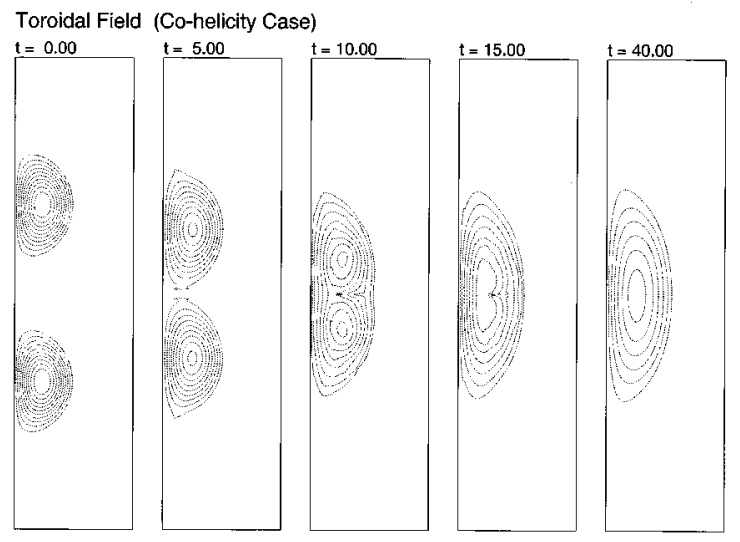

FIG. 7. Same as Fig. 3 but for the co-helicity case.

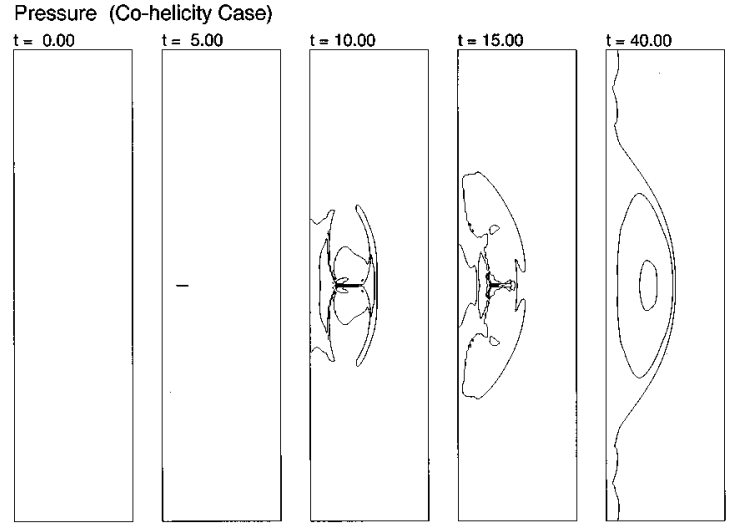

FIG. 8. Same as Fig. 4 but for the co-helicity case.

separatrixes $(\Psi<0)$. As the spheromaks continue to merge, the parallel current that is the dominant component at $t=0$ decreases, but the perpendicular one increases. After the merging, at $t=40 \tau_{\mathrm{A}}$, the perpendicular current has grown about five times larger than the parallel one.

Time evolution of the spatially averaged force $\langle|\mathbf{j} \times \mathbf{B}-\nabla p|\rangle$ is presented in Fig. 5(c), where one can see three major peaks in correspondence to the three peaks of the kinetic energy found in Fig. 5(a). Moreover, the averaged force approaches to zero after the merging. Namely, the MHD equilibrium condition of $\mathbf{j} \times \mathbf{B}=\boldsymbol{\nabla} p$ is nearly satisfied. From the above simulation results, one can conclude that a FRC is spontaneously formed after the counter-helicity merging of spheromaks.

\section{B. An overview of co-helicity merging}

Changing the polarity of the toroidal magnetic field in the lower spheromak, we have carried out a simulation for the co-helicity case. Contour plots of poloidal flux, toroidal field, and pressure for the co-helicity merging are, respectively, given in Figs. 6-8 in the same format as in Figs. 2-4. Also in this case, two spheromaks attract each other and, then, start to merge through the driven reconnection. Opposite to the counter-helicity case, the toroidal field remains after merging. Thus, the final configuration is a spheromak (or a prolomak). This implies that released magnetic energy and thus, the increased thermal energy, in the co-helicity merging should be less than those in the counter-helicity case. The pressure profiles shown in Fig. 8 reflect this fact. A finite pressure gradient is, however, formed even in this case.

Figures 9(a)-(c) show time evolutions of energies, parallel and perpendicular current density, and an averaged force, the same as in Figs. 5(a)-(c). The magnetic energy slightly decreases at about $t=8 \tau_{\mathrm{A}}$ due to the reconnection. After completion of the merging at $t=20 \tau_{\mathrm{A}}$, the magnetic energy continues to decrease owing to the resistive dissipation, while the thermal energy increases gradually. A remarkable difference from the counter-helicity case is found in magnitudes of the parallel and perpendicular currents in Fig. 


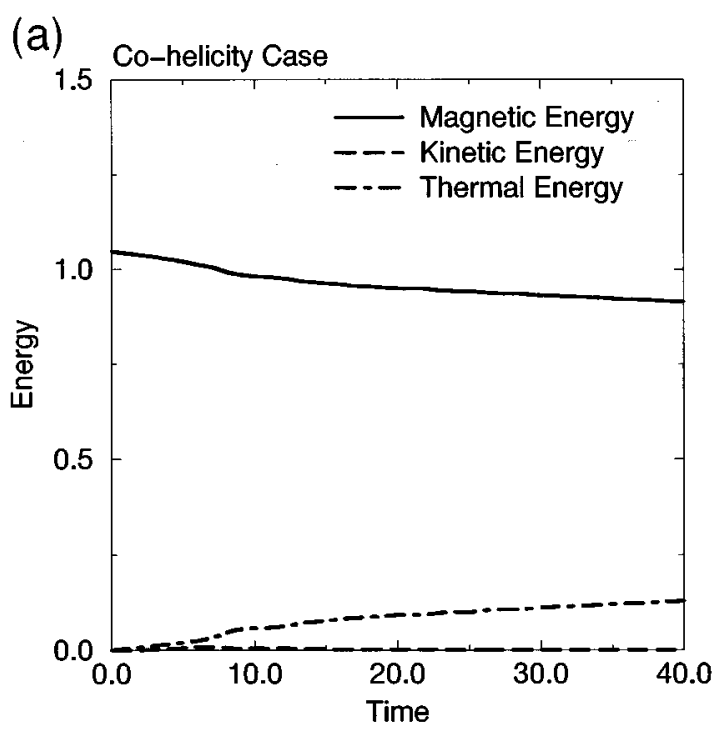

(b)

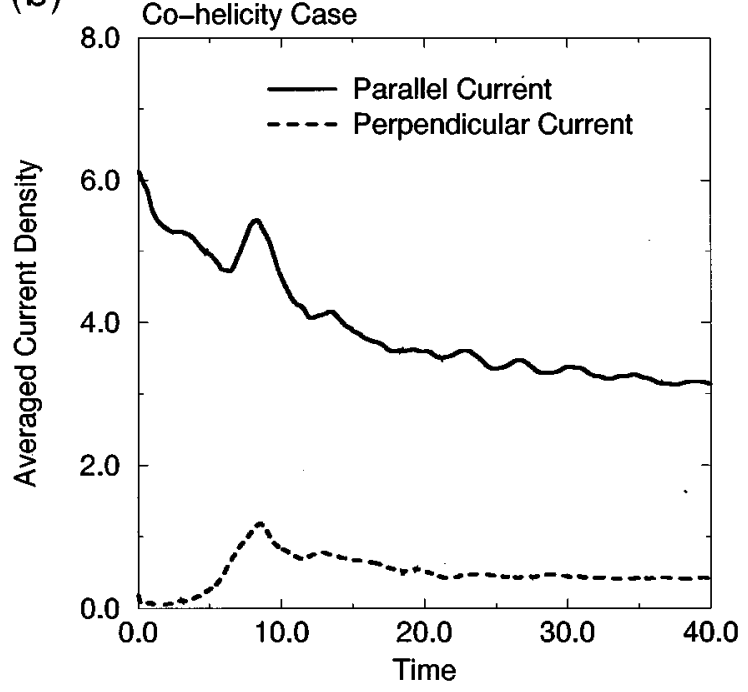

(c)

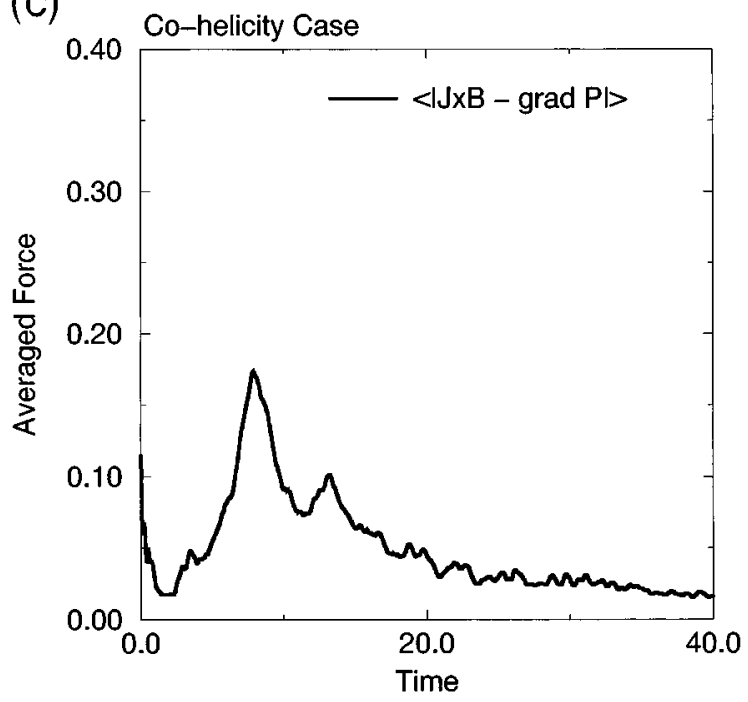

FIG. 9. Same as Fig. 5 but for the co-helicity case.

9(b). Throughout the merging process, the parallel current exceeds the perpendicular component. Thus, the final state is a spheromak type. Nevertheless, the existence of the perpendicular current means that the finite pressure gradient is bal-
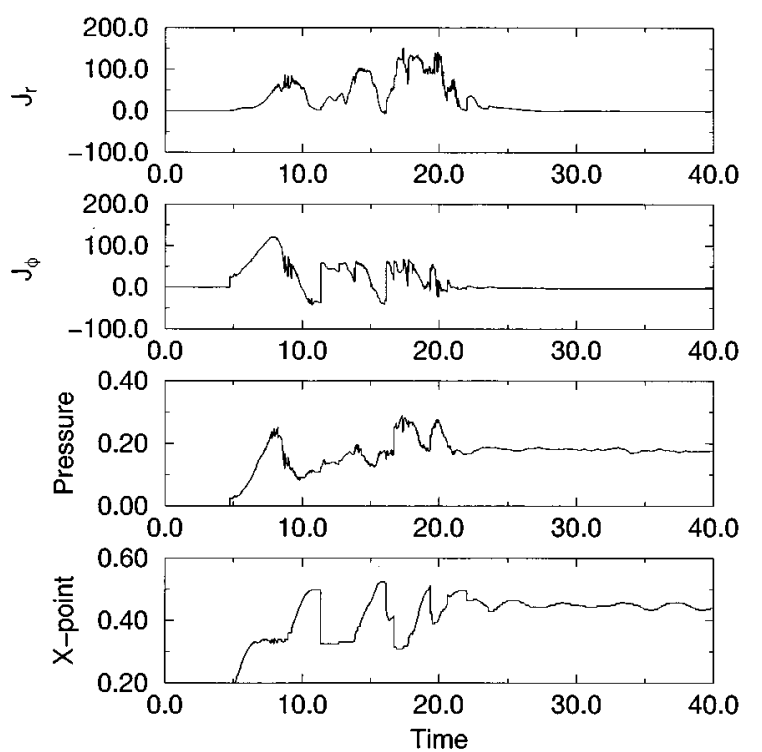

FIG. 10. Temporal plots of the radial and toroidal current density and the pressure observed at the X-point in the counter-helicity merging. The bottom panel shows radial position of the X-point.

anced with the Lorentz force in the final state, as is confirmed in Fig. 9(c).

\section{Finite-pressure effects on magnetic reconnection}

As shown in Fig. 5(a), in the counter-helicity merging, the magnetic energy rather rapidly decreases at $t=8,14$, and $18 \tau_{\mathrm{A}}$. Namely, the magnetic reconnection is enhanced three times, repeatedly. Figure 10 shows temporal plots of three physical quantities measured at the X-point, such as the current density in $r$ and $\varphi$ directions and the pressure. In the bottom panel, the radial position of the X-point is plotted versus time. After completion of the merging, we have picked up data on the magnetic axis. Viewing the plots in Fig. 10, one can see that the relaxation stage of the counterhelicity merging is apparently divided into two phases such as active and inactive ones. In the active phase, the magnetic reconnection is driven by a plasma flow in the $z$ direction, and the peak current intensity at the X-point (reconnection current) is extremely large, more than 100 . Here we remind the reader that the averaged current density at $t=0$ is only 6 as shown in Fig. 5(b). According to the current peaking at the X-point, the pressure is increased by the enhanced Joule heating, while it decreases in the inactive phase. In intervals of the current peaking, namely in the inactive phase, the $r$ (poloidal) component of the reconnection current decreases, and the $\varphi$ (toroidal) component is reversed. This implies not only that the spheromak merging is stopped, but also that the merged flux is re-separated by another reconnection driven in the $r$ direction. In other words, the two spheromaks repeat a bouncing motion.

The time evolution of the current density shown in Fig. 10 clearly reflects the magnetic field configuration of counter-helicity spheromaks. A spheromak has only poloidal field at the edge, while only the toroidal field exists on the magnetic axis. In the initial stage of merging, the poloidal 


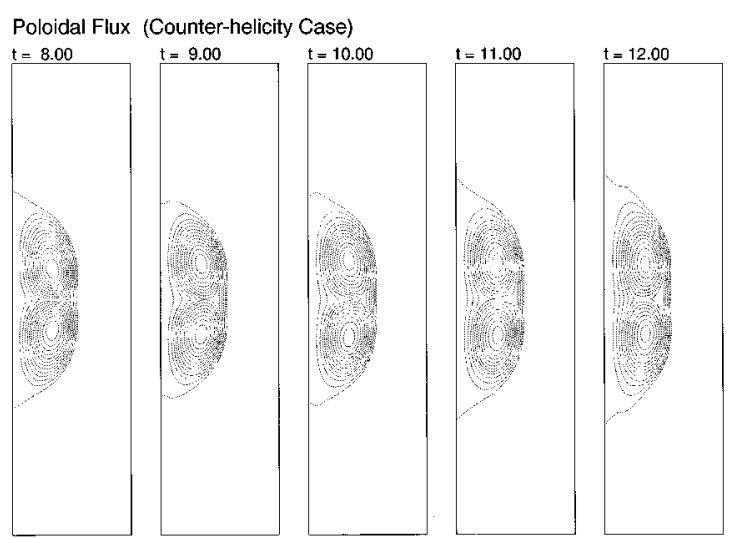

FIG. 11. Same as Fig. 2 but at different time steps.

field dominates in the reconnection process, and thus, the toroidal reconnection current first increases. In the final stage of merging, however, the anti-parallel toroidal field governs the reconnection process, namely, the radial reconnection current becomes much larger than the toroidal one. Incidentally, one can see that, according to radial shift of the $\mathrm{X}$-point, the toroidal current starts to decrease earlier than the radial component. In the active phase, the $\mathrm{X}$-point locates at about $r=0.3$, while it shifts to $r=0.5$ in the inactive phase. When the active phase begins again, the X-point immediately returns to its original position. The shift of the X-point and the decrease of the toroidal reconnection current correspond to shortening of the current sheet between the spheromaks.

In Fig. 11 we have plotted contours of the poloidal flux from $t=8$ to $12 \tau_{\mathrm{A}}$ with high-time resolution. In the first active phase of reconnection at $t=8 \tau_{\mathrm{A}}$, a so-called Y-type current sheet is formed in a contact layer of spheromaks. However, the radial length of the current sheet becomes shorter and shorter, as the spheromaks are slightly bounced back $\left(t=9-10 \tau_{\mathrm{A}}\right)$. At $t=10 \tau_{\mathrm{A}}$, the reconnected flux surfaces are largely bent, and the reconnected flux is accumulated between two spheromaks where one can find a local pressure enhancement (see Fig. 4). Thus, the current sheet shape becomes an X-type. In the second active phase $\left(t=12 \tau_{\mathrm{A}}\right)$, the Y-type current sheet is formed again. The transition of current sheet shape from the Y- to X-type has been found also in a two-dimensional MHD simulation on driven reconnection started from a Harris-type equilibrium. ${ }^{13}$ Thus, the oscillating feature related with the bouncing motion of spheromaks seems to reflect an elementary process of driven reconnection.

The above simulation results suggest that the local pressure enhancement in the vicinity of the $\mathrm{X}$-point plays an essential role in triggering the bouncing motion of spheromaks, while the compressed field repelled the island plasma in the incompressible MHD simulation on coalescence of magnetic islands by Biskamp. ${ }^{14}$ To confirm the effect of pressure, we have also performed three simulation runs changing the value of $\gamma$ from $3 / 2$ to $7 / 3$ for the counterhelicity case. For larger $\gamma$, the reconnection rate will be saturated at a lower level, ${ }^{15}$ since the pressure increases more sensitively to a thermal production rate. Temporal plots of
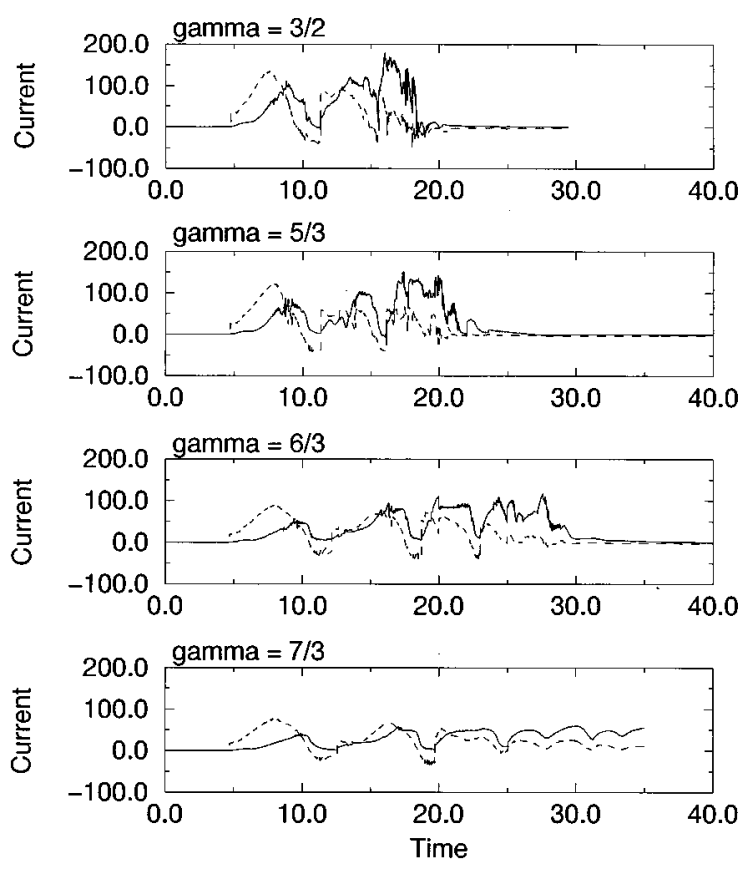

FIG. 12. Temporal plots of current density at the X-point for $\gamma=3 / 2,5 / 3$, $6 / 3$, and $7 / 3$ for the counter-helicity case. Solid and dashed lines show $r$ and $\varphi$ components, respectively.

the reconnection current are given in Fig. 12 for $\gamma=3 / 2$, $5 / 3,6 / 3$, and 7/3. Solid and dashed lines represent $r$ and $\varphi$ components, respectively. One can see that intensity of the reconnection current, namely, the magnetic reconnection rate, largely depends on $\gamma$. For larger $\gamma$, growth of the current density saturates at a lower level, and the merging of spheromaks takes longer. In the case of $\gamma=7 / 3$, the merging has not yet been completed at the end of the simulation $\left(t=35 \tau_{\mathrm{A}}\right)$, although the active phase was repeated six times.

From the above simulation results, we can deduce the following scenario on the dynamic change of the reconnection rate and the bouncing motion of spheromaks. The magnetic reconnection driven by the attracting motion of spheromaks enhances the Joule heating at $z=0$. Then, a highpressure layer is formed between the two spheromaks, while the increased pressure is transported by an outward plasma flow induced by the driven reconnection. If the thermal transport is inefficient with respect to the enhanced Joule heating, the pressure is locally increased more. Hence, a vertical plasma motion is weakened by the local pressure gradients, and the spheromaks are bounced back. Therefore, the reconnection rate is decreased, and the inactive phase begins. When the pressure gradients become sufficiently weak due to the vertical expansion and the convective thermal transport, the reconnection is activated again by the attracting force of spheromaks. Repeating the above process, the reconnection rate changes oscillatory.

The oscillation of the reconnection rate is also found in the co-helicity merging with larger $\gamma$ as shown in Fig. 13. Only the toroidal reconnection current appears in the cohelicity case because of the same toroidal field configuration in the two spheromaks. Also here, as $\gamma$ becomes larger, growth of the reconnection current is suppressed at a lower 

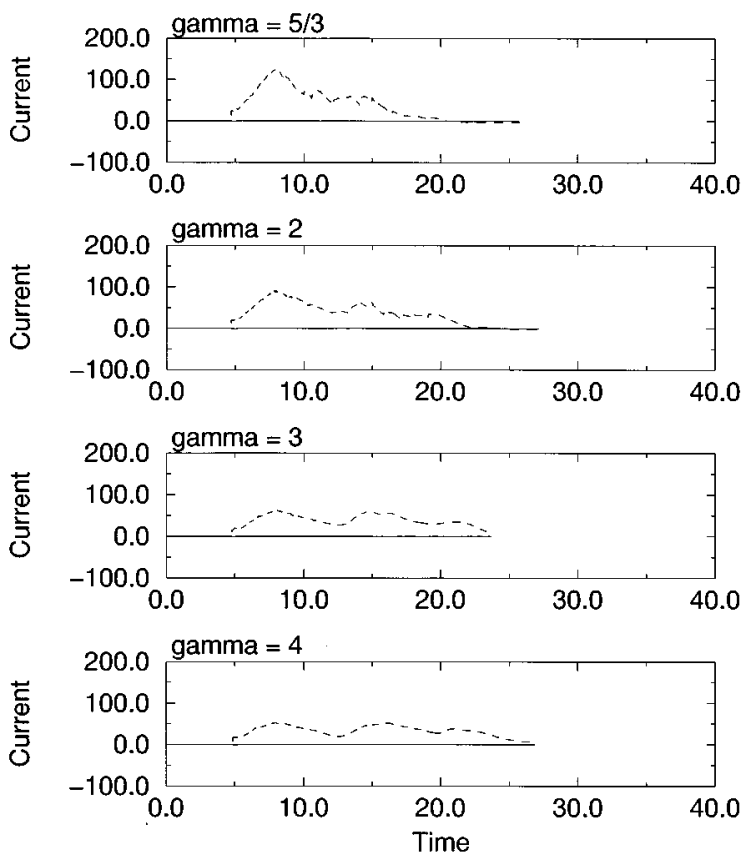

FIG. 13. Same as Fig. 12 but for the co-helicity merging with $\gamma=5 / 3,2$, 3 , and 4 .

level, and the oscillation becomes clearer. For the cases of $\gamma=3$ and 4 , the active phase is repeated three times.

The above simulation results support our scenario that the local pressure enhancement causes the decrease of the reconnection rate and, exciting the bouncing motion of spheromaks, prevents progress of the merging.

\section{Spontaneous pressure confinement in the FRC}

In the counter-helicity case, the global pressure profile of which gradient balances with the Lorentz force is spontaneously formed, as a natural consequence of the spheromak merging. A formation process of the pressure profile is described in this section. Figure 14 shows time evolutions of pressure for the counter-helicity case observed at five different points, $r=0.3$ and $z=0.6,0.45,0.3,0.15$, and 0 , respectively. Here, $\gamma=5 / 3$. Corresponding to the start of reconnection, the pressure is increased at $z=0$ by the Joule heating. After a while, at $t=12 \tau_{\mathrm{A}}$, the pressure rises at $z=0.6$, although it is away from the X-point. As the observation point approaches the X-point, except $z=0$, pressure increases later and later. Hence, as shown in Fig. 4, a hollow pressure profile is temporarily formed.

A probable mechanism to explain the formation of the pressure profile is thermal transport due to a poloidal flow, namely, convective transport and compressive heating. The driven reconnection induces a poloidal flow which diverges from the X-point and can transport the increased thermal energy. If the poloidal flow is parallel to flux surfaces, the pressure will increase along the reconnected flux surfaces due to the convection and/or the compression. As the merging proceeds, the poloidal flow makes the plasma heating along the inner flux surfaces. Moreover, the compressive heating can adjust the pressure profile so that the pressure
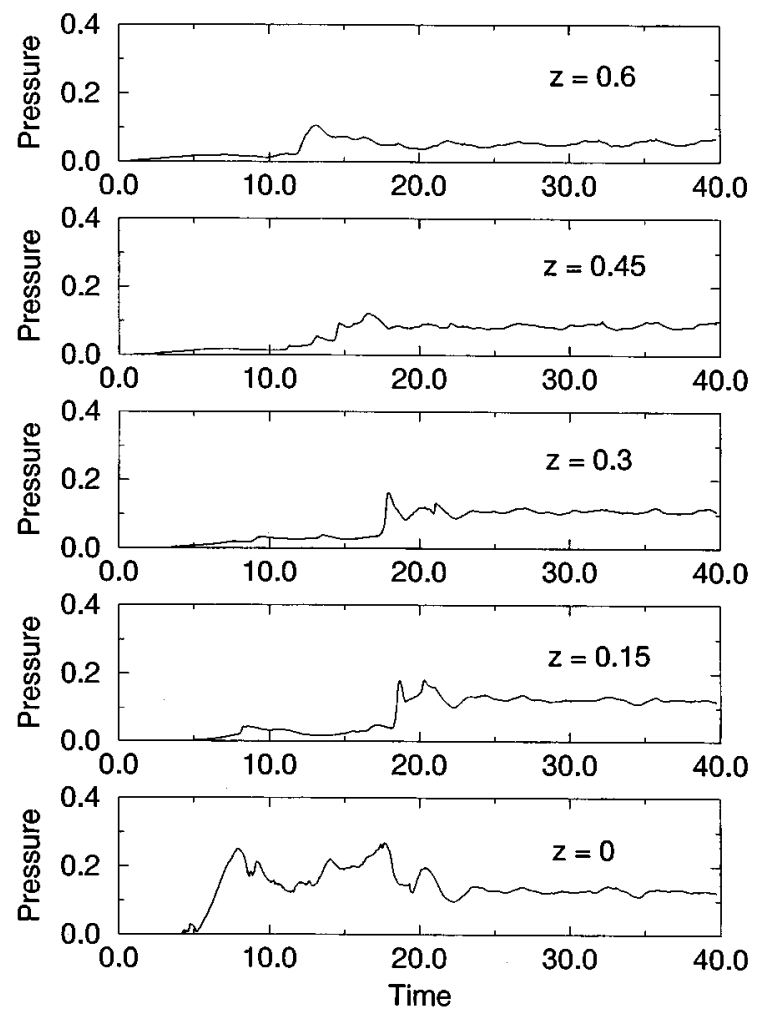

FIG. 14. Time evolution of the pressure observed at $r=0.3$ and $z=0.6$, $0.45,0.3,0.15$, and 0 in the counter-helicity case.

gradient balances with the Lorentz force. Therefore, the pressure profile, of which iso-surfaces coincide with the flux surfaces, can be generated spontaneously.

As explained in detail in the next section, in the counterhelicity merging a reconnected magnetic loop oscillates in the toroidal direction, relaxing excessive magnetic tension and accelerating a toroidal flow. Thus, according to the toroidal oscillation, the magnetic field is sheared between reconnected and unreconnected flux surfaces, where a sheet current is induced. The Joule heating due to the sheet current and the viscous damping of the toroidal flow also contribute to increase the thermal energy. Nevertheless, the thermal transport of a poloidal flow is indispensable to equalize the pressure along flux surfaces.

To check the above hypothesis, we have plotted poloidal flow patterns at different time steps in Fig. 15. At $t=5 \tau_{\mathrm{A}}$, a plasma flow toward $z=0$ starts to make the spheromaks merge. In the merging phase, at $t=10 \tau_{\mathrm{A}}$, the flow pattern is rather complicated. Comparing Fig. 15 with Fig. 2, one can find a poloidal flow component around the unmerged flux surfaces. At $t=15 \tau_{\mathrm{A}}$, the poloidal flow is generated around inner flux surfaces, as the merging proceeds deeper. Figure 16 shows historical plots of the pressure and its source terms observed at $r=0.3$ and $z=0.6$. In the top panel, time evolution of the pressure is replotted for a reference. Convection, $-\mathbf{v} \cdot \boldsymbol{\nabla} p$, compression, $-\gamma p \boldsymbol{\nabla} \cdot \mathbf{v}$, Joule heating, $(\gamma-1) \eta \mathbf{j}^{2}$, and viscous heating, $(\gamma-1) \Phi$, terms are respectively shown in Fig. 16. As expected, both the convection and compression terms are enhanced according to the increase of pressure. The Joule and viscous heating terms are minor compo- 


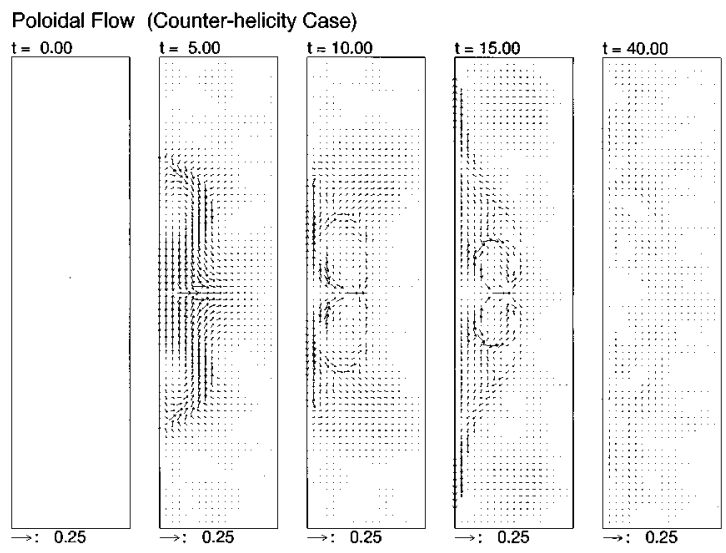

FIG. 15. Poloidal flow patterns at different time steps in the counter-helicity merging.

nents. Therefore, it is concluded that the pressure profile is spontaneously generated by the thermal transport of the poloidal flow induced by the driven reconnection.

\section{E. Toroidal oscillation of a magnetic loop}

During the counter-helicity merging of spheromaks, the toroidal field is reversed on reconnected flux surfaces (see Fig. 3). A mechanism of the field reversal has been considered as follows. ${ }^{1,4}$ Because of the counter-helicity configuration with the anti-parallel toroidal field, a just-reconnected
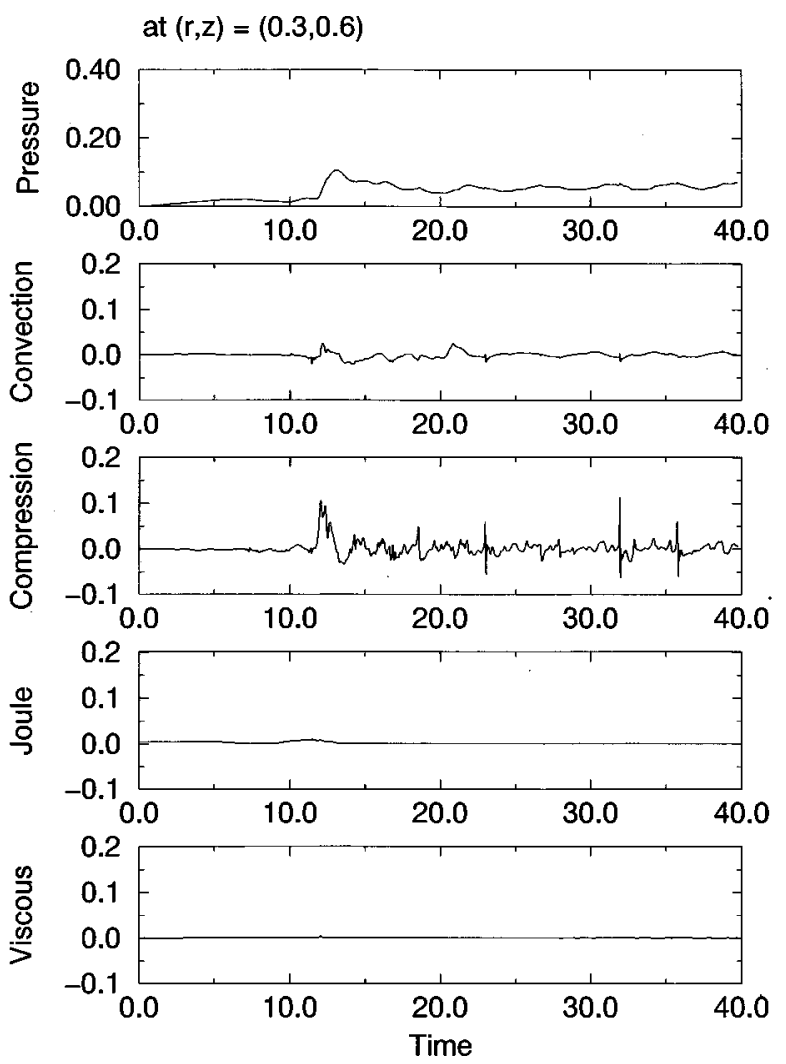

FIG. 16. Temporal plots of the pressure, convection, $-\mathbf{v} \cdot \boldsymbol{\nabla} p$, compression, $-\gamma p \boldsymbol{\nabla} \cdot \mathbf{v}$, Joule heating, $(\gamma-1) \eta \mathbf{j}^{2}$, and viscous heating, $(\gamma-1) \Phi$, terms at $r=0.3$ and $z=0.6$ in the counter-helicity case.

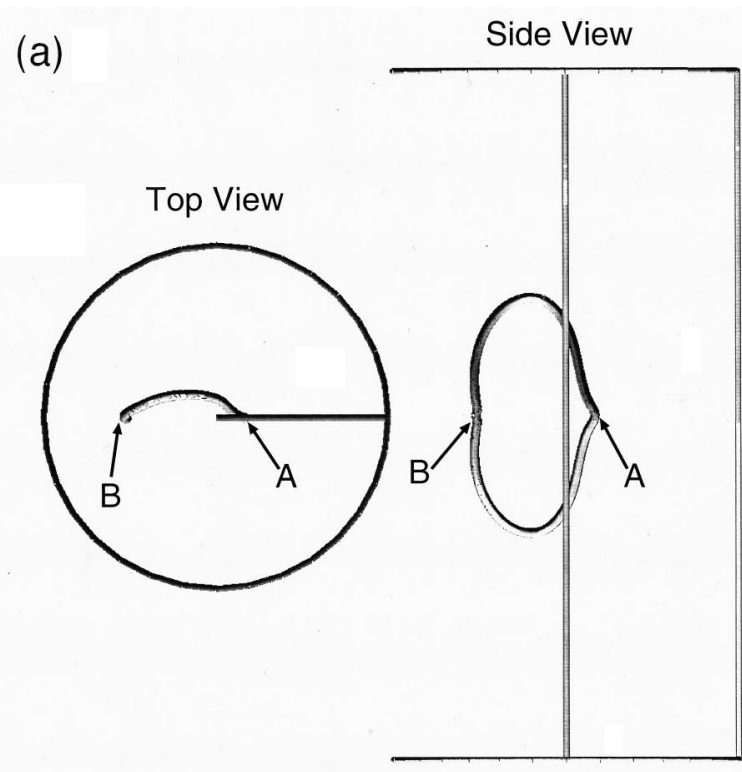

(b)

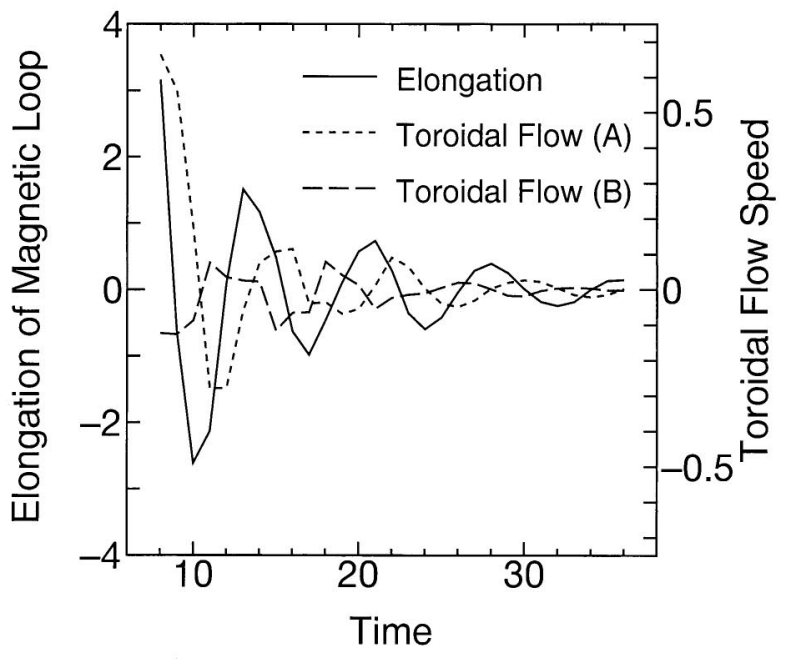

FIG. 17. Dynamic behavior of a reconnected magnetic loop in the counterhelicity merging. (a) Three-dimensional plots of the magnetic loop of $\Psi=-5 \times 10^{-3}$ in the cylindrical vessel at $t=8 \tau_{\mathrm{A}}$. (b) Historical plots of the elongation of the magnetic loop defined by the relative toroidal angle $\varphi_{B}-\varphi_{A}$ and the toroidal flow velocity at points $A$ and $B$.

magnetic loop is inclined with respect to a poloidal plane, in other words, elongated in toroidal direction. The elongated magnetic loop causes strong acceleration of a toroidal flow (a slingshot effect). If the toroidal flow speed is sufficiently large, the magnetic loop may overshoot an equilibrium position. Therefore, the toroidal field is reversed and the magnetic loop oscillates in the toroidal direction (Alfvén oscillation).

In Fig. 17(a), we have plotted a magnetic loop of $\Psi=-5 \times 10^{-3}$ which is just-reconnected at $t=8 \tau_{\mathrm{A}}$. One can see that the magnetic loop is actually elongated in $\varphi$ direction, and that its configuration is quite different from the FRC with no toroidal field. Thus, excessive tension of the magnetic loop should be relaxed, and the plasma is accelerated in toroidal direction. In the relaxation process of the 
excessive magnetic tension, therefore, one will find the reversal of toroidal field.

To examine the toroidal oscillation of the magnetic loop, we have traced the motion of two representative points on the loop which are marked by A and B in Fig. 17(a). The solid line in Fig. 17(b) indicates elongation of the magnetic loop defined by a relative toroidal angle of the two points, A and $\mathrm{B}$, that is, $\varphi_{B}-\varphi_{A}$. Toroidal oscillation of the magnetic loop is clearly found in the figure. When the elongation angle becomes negative, the toroidal field is reversed. The oscillation is gradually damped by the finite resistivity and viscosity. Then, the elongation angle approaches zero, namely, the toroidal field vanishes along the magnetic loop. Dotted and dashed lines in Fig. 17(b), respectively, show toroidal flow speed at points $A$ and $B$. The toroidal flow at the point $A$ is strongly accelerated up to $0.7 V_{\mathrm{A}}$, where $V_{\mathrm{A}}$ is the typical Alfvén speed. Corresponding to the field line motion, the toroidal flow changes its direction because of the slingshot effect. Oscillations found in the flow speed lead by $\pm \pi / 2$ in phase to that of the magnetic loop. Thus, it is summarized that the Alfvén coupling of the magnetic loop motion and the toroidal flow causes the toroidal acceleration and the field reversal.

\section{F. Effects of parallel thermal conduction}

In real plasmas, heat transport of an electron flow parallel to the magnetic field is considered to be more efficient than that of the bulk plasma flow, because of a fast electron motion along field lines. The electron heat transport would cause the thermal energy diffusion along field lines through some kinds of wave-particle interactions. Thus, it is expected that the "anomalous" parallel thermal conduction could be much larger than other diffusion processes such as the perpendicular conduction and the resistive or viscous dissipations. While no thermal conduction is considered in the previous sections, therefore, we assume an "infinitely large", parallel thermal conduction effect as another limiting case.

To properly include the thermal conduction, it is necessary to calculate the density change by solving the equation of continuity,

$$
\frac{\partial \rho}{\partial t}=-\nabla \cdot(\rho \mathbf{v})
$$

Thus, to resolve a highly compressed current sheet, we have adopted an inhomogeneous grid system in the $z$ direction with the minimum grid spacing of $1 \times 10^{-3}$. Total grid points are $401 \times 473$ in $r-z$ plane.

The "infinitely large", parallel thermal conduction is introduced by taking the flux-surface average of temperature at every simulation time step. The simulation procedure is summarized as follows. Along with Eqs. (1), (3), and (13), we calculate increment of pressure $\delta p$ in a time step by the energy equation, Eq. (2). Then, the temperature averaged in the flux surfaces, $\overline{T^{n+1}}$, is obtained by $\overline{T^{n+1}}=\overline{\left(p^{n}+\delta p\right)} / \overline{\rho^{n+1}}$, where the superscript $n$ denotes the simulation step. The upper bar means the flux-surface average. Pressure at the $n+1$ time step is, therefore, given by $p^{n+1}=\rho^{n+1} \overline{T^{n+1}}$ so that the thermal energy is conserved (a)

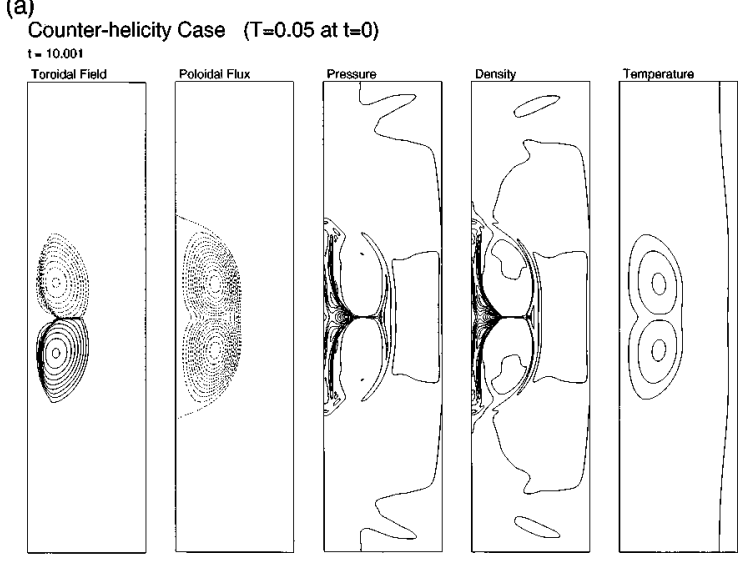

(b)

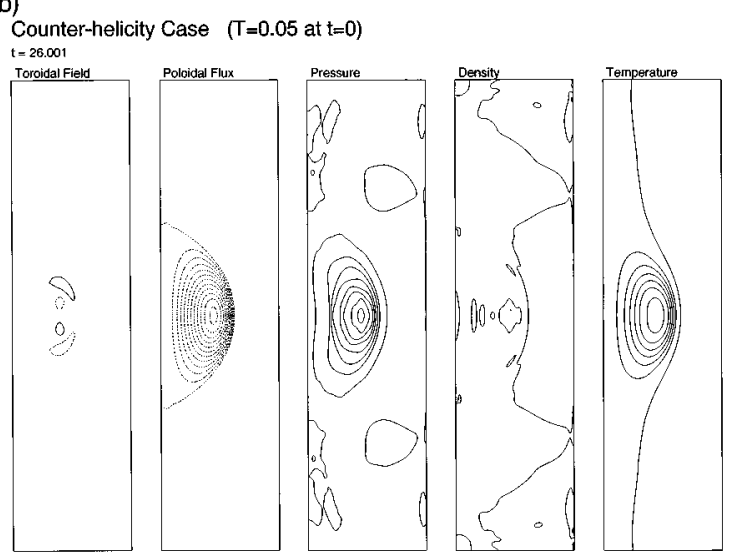

FIG. 18. Contour plots of toroidal field, poloidal flux, pressure, density and temperature for the counter-helicity merging with "infinitely large" parallel thermal conductivity at (a) $t=10$ and (b) $26 \tau_{\mathrm{A}}$.

along each flux surface throughout this procedure. The above method would give a proper result when the time step is sufficiently smaller than a typical time scale of pressure such as the transit time of the fast magnetosonic wave. For a convergence check we have done a simulation with a half time step and have obtained the same result.

Setting up the same initial condition with the previous sections, except for the initial temperature $T=0.05$, we have performed simulations of the co- and counter-helicity merging. Figure 18(a) shows snapshots of toroidal field, poloidal flux, pressure, density and temperature at $t=10 \tau_{\mathrm{A}}$ for the counter-helicity case. It should be noted that the pressure sharply increases in the reconnection layer, although the temperature is equalized along the flux surfaces. This is because the plasma is strongly compressed by the vertical motion of spheromaks. Then, the pressure increases so much as well as the plasma density. A peak value of the density reaches 3.25. Reflecting the plasma compression, the density contours coincide well with the pressure profile.

As the counter-helicity merging proceeds, the pressure and density profiles are spontaneously organized toward the finial equilibrium state. We have plotted snapshots at $t=26 \tau_{\mathrm{A}}$ in Fig. 18(b) where one can see a FRC without the mean toroidal field. In the final state, the pressure gradient nearly balances with the Lorentz force. Thus, the pressure 
contours become similar to the poloidal flux ones. Interestingly, the density profile is flattened due to the convectional transport, while it peaked in the reconnection layer at $t=10 \tau_{\mathrm{A}}$. Therefore, not only the thermal energy transport but also the mass transport due to the plasma flow plays an important role in the formation process of FRC.

The local pressure enhancement near the reconnection point causes the bouncing motion of spheromaks even in the present simulation with the parallel thermal conduction. Actually, we have observed the bouncing motion three times. Thus, the counter-helicity merging takes a longer time $\left(\sim 25 \tau_{\mathrm{A}}\right)$ than the co-helicity one $\left(\sim 18 \tau_{\mathrm{A}}\right.$; not shown). For comparison with this result, we have also carried out simulations with initial uniform temperature $T=0.05$ using the same simulation model given in the previous sections (without thermal conduction and density change), and found that the counter-helicity merging took more time $\left(\sim 30 \tau_{\mathrm{A}}\right)$ while the co-helicity merging finished at $t \sim 22 \tau_{\mathrm{A}}$. Therefore, it is considered that the local pressure enhancement by the compression and the Joule heading reduces the merging speed and causes the bouncing motion, although the parallel thermal conduction certainly hastens the merging.

One may consider that the density, and also the pressure, should be equalized along field lines as well as the temperature. However, introducing the parallel thermal conduction effect, we have assumed a fast electron motion along field lines, while the density profile is predominantly determined by the ion dynamics. Thus, the density and pressure inhomogeneity along field lines cannot be relaxed within the compressible MHD time scale, namely, it excites the magnetosonic waves. In longer time scales, the density and pressure will be uniform along field lines as seen in the final state of the present simulation [Fig. 18(b)], because of damping of the magnetosonic waves. Generation of the density and pressure inhomogeneity is, thus, an intrinsic feature of the compressible MHD phenomena.

\section{SUMMARY AND DISCUSSION}

We have investigated the coalescence process of two spheromaks for the co- and counter-helicity cases, employing the axisymmetric MHD simulation code. Obtained results are summarized as follows:

(1) In the counter-helicity merging of low- $\beta$ spheromaks, a FRC is formed through the driven magnetic reconnection which locally increases the thermal energy in the vicinity of the X-point, while a larger spheromak (or a prolomak) is obtained in the co-helicity case.

(2) The increased thermal energy is transported by the poloidal flow which is generated by the driven reconnection. Hence, the pressure profile of which iso-surfaces coincide with the flux surfaces is spontaneously formed, even if the parallel thermal conduction effect is not taken into account. In the final state of the counter-helicity merging, most of the increased thermal energy is confined in the closed flux surfaces.

(3) The driven magnetic reconnection is repeatedly enhanced in the merging process, according to the bouncing motion of spheromaks. During the bouncing motion triggered by the local pressure enhancement, the current sheet shape changes from the Y- to X-type.

(4) The toroidal Alfvén oscillation of a reconnected magnetic loop is clearly demonstrated in the counter-helicity case. The toroidal field around the unmerged flux surfaces is temporarily reversed by the accelerated toroidal flow, as shown by the TS-3 experiments.

(5) We have also performed simulations with the parallel thermal conduction effect. Even in this case, the local increase of pressure in the reconnection layer due to the compression and the Joule heating causes the bouncing motion and the reduction of the reconnection rate.

(6) Because of the compression, the plasma density is also increased in the reconnection layer during the spheromak merging with the thermal conduction effect. The density profile is, however, flattened by the mass transport of the poloidal flow, and then, becomes nearly uniform after merging.

The present simulation results are qualitatively consistent with the TS-3 experiments for the formation of the FRC, the reversal of toroidal field, and the acceleration of toroidal flow. The bouncing motion of spheromaks is, however, not clearly found in the counter-helicity merging experiments. Moreover, experimental estimation of the reconnection rate in the counter-helicity case is about three times higher than that in the co-helicity one, ${ }^{1-5}$ while in the present simulation the co-helicity merging has been finished earlier than the counter-helicity case. These discrepancies between the present simulations and the experiments may originate from differences in viscosity, resistivity, and non-MHD (two-fluid and kinetic) effects. In the following, we will discuss these issues in detail.

In order to examine an effect of the viscosity, we have performed two additional simulation runs with $\mu=3 \times 10^{-4}$ and $1 \times 10^{-3}$. In the case of $\mu=1 \times 10^{-3}$, the oscillation of the reconnection rate does not clearly appear, and the current density at the X-point slowly evolves in time. On the other hand, the bouncing motion is repeated three times for $\mu=3 \times 10^{-4}$. Although the present viscosity model is much simpler than that in real plasmas, it seems that for occurrence of the bouncing motion the viscosity is needed to be as weak as a flow pattern nearby the X-point is changed by the local pressure enhancement.

In our simulations, the local pressure enhancement reduces a speed of the counter-helicity merging. In actual experiments, however, electrons lose their kinetic energy by radiative cooling because of the low electron temperature $(\sim 10 \mathrm{eV}) .{ }^{1}$ The cooling effect would hasten the merging, since the pressure profile could not be peaked in the vicinity of the X-point. If we incorporate the electron cooling effect into our simulation model, the reconnection current sheet should be much thinner than that in the present ones. Then, the counter-helicity merging might be faster than the cohelicity case, since the toroidal field compressed in the very thin current sheet between the co-helicity spheromaks would repel the plasma flow toward the X-point as suggested by Sato et al. ${ }^{15}$ However, thickness of the current sheet observed in the TS-3 experiments is estimated as $1-5 \mathrm{~cm}$, 
which is longer than that of the present simulations. It implies, therefore, that the cooling effect does not play a leading role in the faster counter-helicity merging, and that nonMHD effects, rather than the classical resistivity and the pressure gradient, would determine the thickness of the current sheet in the TS- 3 merging experiments.

In the TS-3 experiments, ${ }^{4}$ moreover, the "anomalous" resistivity dominates in the counter-helicity merging, and largely enhances the reconnection rate. The estimated resistivity in the counter-helicity case becomes $5-7$ times larger than that in the co-helicity one. It is considered that the "anomalous" resistivity is caused by two-fluid and/or kinetic effects of plasmas, such as the electron inertia, Hall term, and particle orbit effects. It has also been quantitatively pointed out that the collisional resistivity can not be responsible for the reconnection rate in the fast sawteeth crash in large tokamaks. ${ }^{16}$ Employing the generalized Ohm's law with the electron inertia and pressure gradient terms, Aydemir found fast growth of a collisionless $m=1$ mode in a high-temperature tokamak plasma. ${ }^{17}$ The magnetic Reynolds number in the TS-3 experiments is, however, estimated to be as small as several hundreds ${ }^{1}$ because of the low electron temperature, while it is larger than $10^{8}$ in large tokamaks. In addition, the electron skin depth $c / \omega_{p e}$ in the TS-3 plasma with the number density of $1 \times 10^{20} \mathrm{~m}^{-3}$ is about $0.5 \mathrm{~mm}$, and is much shorter than the typical thickness of the current sheet. Thus, the Ohm's law given by Eq. (5) seems to be a good approximation to the TS-3 plasma. Biskamp et al. ${ }^{18}$ have shown that, decoupling the electron and ion motions in a strong longitudinal magnetic field, the Hall term enhances the reconnection rate on scales smaller than the ion skin depth $c / \omega_{p i}$. The effect of the Hall term, however, should be minor in the counter-helicity case where the magnetic field vanishes at the reconnection point. The other non-MHD effect which may dominate in the TS-3 experiments is the particle orbit effect. Because of no magnetic field at the $\mathrm{X}$-point in the counter-helicity case, an effect of meandering particle orbit would play an important role in production of the "anomalous" resistivity. Actually, it is found in recent TS-3 experiments that the "anomalous" resistivity appears when the thickness of the current sheet becomes shorter than the ion gyroradius. ${ }^{19}$ Introduction of the particle orbit effect into the MHD model is an important but a difficult problem, and is beyond the scope of the present paper. Recently, Horiuch and Sato studied the collisionless driven reconnection process by means of the particle simulation, ${ }^{20,21}$ and found that the reconnection mechanism changed from a particle meandering dominance in a weak longitudinal magnetic field to an electron inertia dominance in a strong field. They also confirmed that, being independent of the longitudinal mag- netic field, the reconnection rate was determined by a flux input rate into the system, that is, an imposed longitudinal electric field. ${ }^{21}$ Therefore, the faster counter-helicity merging found in the experiments remains to be solved in future studies.

In this study, we have assumed the axisymmetry, and have carried out the two-dimensional simulations. To discuss about the minimum energy state after the coalescence of spheromaks, however, we should consider all of the ideal instabilities in a full three-dimensional system. Although the $n=1$ tilt and/or shift modes may be unstable in the threedimensional system, it could be stabilized by a center conductor. ${ }^{22}$ We will also investigate a three-dimensional effect on the spheromak merging in the future.

\section{ACKNOWLEDGMENTS}

One of the authors (T.-H.W.) would like to thank M. Yamada, R. M. Kulsrud, T. Ohkawa, L. Steinhauer, A. Ishida, Y. Ono, M. S. Chu, S.-P. Zhu, and H. Kitabata for stimulating discussions and fruitful comments. Numerical computations in this study are performed on the NIFS Advanced Computing System for Complexity Simulation.

${ }^{1}$ M. Yamada, Y. Ono, A. Hayakawa, M. Katsurai, and F. W. Perkins, Phys. Rev. Lett. 65, 721 (1990).

${ }^{2}$ M. Yamada, F. W. Perkins, A. K. MacAulay, Y. Ono, and M. Katsurai, Phys. Fluids B 3, 2379 (1991).

${ }^{3}$ Y. Ono, A. Morita, T. Itagaki, and M. Katsurai, Plasma Phys. Controlled Nucl. Fusion Res. 1992, 619 (1993).

${ }^{4}$ Y. Ono, A. Morita, M. Katsurai, and M. Yamada, Phys. Fluids B 5, 3691 (1993).

${ }^{5}$ Y. Ono, Trans. Fusion Tech. 27, 369 (1995).

${ }^{6}$ J.B. Taylor, Phys. Rev. Lett. 33, 1139 (1974)

${ }^{7}$ J.B. Taylor, Rev. Mod. Phys. 58, 741 (1986)

${ }^{8}$ T. Sato, Y. Oda, S. Otsuka, K. Katayama, and M. Katsurai, Phys. Fluids 26, 3602 (1983).

${ }^{9}$ B. Dasgupta, T. Sato, T. Hayashi, K. Watanabe, and T.-H. Watanabe, Trans. Fusion Tech. 27, 374 (1995).

${ }^{10}$ T.-H. Watanabe, T. Sato, T. Hayashi, and K. Watanabe, J. Plasma Fusion Res. 72, 101 (1996) (in Japanese).

${ }^{11}$ M. N. Rosenbluth and M. N. Bussac, Nucl. Fusion 19, 489 (1979).

${ }^{12}$ T. Sato and T. Hayashi, Phys. Fluids 22, 1189 (1979).

${ }^{13} \mathrm{H}$. Kitabata, Ph.D thesis, The Graduate University for Advanced Studies, Japan, 1996.

${ }^{14}$ D. Biskamp, Phys. Rep. 4, 179 (1994), and references therein.

${ }^{15}$ T. Sato, T. Hayashi, K. Watanabe, R. Horiuchi, M. Tanaka, N. Sawairi, and K. Kusano, Phys. Fluids B 4, 450 (1992).

${ }^{16}$ J. A. Wesson, Plasma Phys. Controlled Nucl. Fusion Res. 1990, 79 (1991);

J. F. Drake and R. G. Kleva, Phys. Rev. Lett. 66, 1458 (1991).

${ }^{17}$ A. Y. Aydemir, Phys. Fluids B 4, 3469 (1992).

${ }^{18}$ D. Biskamp, E. Schwarz, and J. F. Drake, Phys. Rev. Lett. 75, 3850 (1995).

${ }^{19}$ Y. Ono (private communication, 1996).

${ }^{20}$ R. Horiuchi and T. Sato, Phys. Plasmas 1, 3587 (1994).

${ }^{21}$ R. Horiuchi and T. Sato, Phys. Plasmas 4, 277 (1997).

${ }^{22}$ T. Hayashi and T. Sato, Phys. Fluids 28, 3654 (1985). 